\title{
Erratum: Additive interfacial chiral interaction in multilayers for stabilization of small individual skyrmions at room temperature
}

C. Moreau-Luchaire, C. Moutafis, N. Reyren, J. Sampaio, C. A. F. Vaz, N. Van Horne, K. Bouzehouane, K. Garcia, C. Deranlot, P. Warnicke, P. Wohlhüter, J.-M. George, M. Weigand, J. Raabe, V. Cros and A. Fert

Nature Nanotechnology 11, 444-448 (2016); published online 18 January 2016; corrected online 23 March 2016; corrected after print 20 July 2016

In the version of this Article originally published, the equation ' $W=(1 / 4 \pi) \int \mathbf{s} \cdot\left(\partial_{x} \mathbf{s} \times \partial_{y} \mathbf{s}\right) \mathrm{d} x \mathrm{~d} y$ ' was missing the symbol for the vector product and the first erratum did not correct the problem. Further, unrelated, changes were also required: in the sentence beginning 'We present next the micromagnetic simulations..., 'ten' should have been 'eleven', and in the sentence beginning 'With the smallest exchange constant..., ' $1.6 \mathrm{pJ} \mathrm{m}^{-1}$ ' should have read ' $1.6 \mathrm{~mJ} \mathrm{~m}^{-2}$. All of these errors have now been corrected in the online versions of the Article. 